\title{
The Effects of Foot Reflexology on the Physical Symptoms of Cancer Patients
}

\author{
Young-Ran Yeun $\mathrm{PhD}^{1}$, Yi-Sub Kwak $\mathrm{PhD}^{2, \dagger}$, Hye-Young Kim $\mathrm{PhD}^{3, \dagger}$ \\ ${ }^{1}$ Department of Nursing, Kangwon National University, Samcheok; ${ }^{2}$ DEU-Exe-Physio Lab, Department of Physical Education, College of Arts and Sports Science, Dong- \\ Eui University, Busan; ${ }^{3}$ Department of Dental Hygiene, Kangwon National University, Samcheok, Korea
}

PURPOSE: Foot reflexology has become popular for sustainable health management because it is free from side effects, easy to learn and perform. The purpose of this study was to investigate the effects of foot reflexology on the physical symptoms of cancer patients.

METHODS: The systematic review and meta-analysis was conducted by following the Preferred Reporting Items for Systematic Reviews and Meta-Analyses (PRISMA). Data were pooled using fixed- or random-effects models. Pain, sleep, fatigue, and nausea and vomiting were analyzed for the main effects. Eighteen studies were included, representing 931 participants.

RESULTS: Foot reflexology was effective in alleviating pain $(\mathrm{d}=-1.05 ; 95 \% \mathrm{CI}$ : $-1.52,-0.59)$, promoting sleep $(\mathrm{d}=1.15$; $95 \% \mathrm{CI}$ : 0.45 , 1.85), and relieving fatigue ( $\mathrm{d}=-1.46 ; 95 \% \mathrm{CI}:-1.79,-1.14)$, but it was not significantly effective in reducing nausea and vomiting $(\mathrm{d}=-$ 0.53 ; $95 \%$ CI: $-1.29,0.22)$.

CONCLUSIONS: Based on these findings, there is clear evidence that foot reflexology has significant positive effects on the physical symptoms of cancer patients. Further study is needed, however, to perform subgroup analysis using the frequency of sessions as predictors.

Key words: Cancer, Fatigue, Foot reflexology, Pain, Sleep

\section{INTRODUCTION}

Despite the advances in modern medicine, the incidence of cancer, the no. 1 cause of death in South Korea at present, has increased by about 3.3\% annually over the past decade. For the South Koreans who meet the current life expectancy rate, cancer is estimated to occur in 2 out of 5 men (38.3\%) and in 1 out of 3 women (33.3\%) [1].

Cancer patients experience lots of physical pains during cancer diagnosis and treatment. The main symptoms patients complain of include fatigue, anorexia, pain, sleep disorders, and nausea and vomiting [2,3]. In particular, these symptoms are characterized by clustering, in which several symptoms occur simultaneously or sequentially rather than alone
[4]. According to previous relevant studies, these symptoms have been reported to affect the disease recovery of cancer patients due to the nature of recurring diseases $[5,6]$. Therefore, appropriate interventions are needed to control the physical problems of cancer patients so as to enhance their daily life adaptation and improve their quality of life.

Many cancer patients worldwide use a variety of complementary and alternative medicines (CAMs) to relieve symptoms. National Cancer Institute (NCI) in the U.S. provides practical guidance by investigating evidence-based CAMs for the control and treatment of major symptoms experienced by cancer patients [7]. In South Korea, more than $42 \%$ of the cancer patients reported that they had an experience of using CAMs [8]. Foot reflexology uses the toes to stimulate the acupressure points

Corresponding author: Yi Sub Kwak Tel +82-51-890-1546 Fax +82-505-182-6915 E-mail ysk2003ddeu.ac.kr

Corresponding author: Hye-Young Kim Tel +82-33-540-3392 E-mail khy0606a kangwon.ac.kr

${ }^{\dagger}$ These authors contributed equally to conduct of the studies.

Keywords Cancer; fatigue; foot reflexology; pain; sleep

Received 23 Nov 2020 Revised 13 Jan 2020 Accepted 28 Jan 2020

(a)This is an Open Access article distributed under the terms of the Creative Commons Attribution Non-Commercial License (https://creativecommons.org/licenses/by-nc/4.0/) which permits unrestricted non-commercial use, distribution, and reproduction in any medium, provided the original work is properly cited. 
corresponding to each part of the body, reducing the tension of the skin and muscles and improving circulation [9]. It can be using holistic health approach that reduce the severity of chemotherapy-induced vomiting and nausea of some kinds of cancer patients.

Foot reflexology has been proven to be effective in cancer patients' symptom management with only one application, and many interventional studies have been conducted since it was reported to be effective in enhancing adaptability in daily life and improving the quality of life [10-12]. Accordingly, systematic reviews of foot reflexology were conducted, but these studies included subjects other than cancer patients [13] or other CAMs $[14,15]$. There have been few studies that systematically consider the effects of foot reflexology in cancer patients. Therefore, this study aimed to provide fundamental data for evidence-based nursing practice by systematically examining and analyzing the effects of foot reflexology intervention applied to cancer patients on their physical symptoms.

\section{METHODS}

\section{Eligibility Criteria}

The study selection criteria is based on PICOTS-SD (Participants, Intervention, Comparisons, Outcomes, Timing of Outcome Measurement, Settings, Study Design): participants of this study $(\mathrm{P})$ were cancer patients; method of intervention (I) was foot reflexology; comparison group (C) was comprised of those not undergoing interventions or programs comparable to the main one; outcome of the study $(\mathrm{O})$ were pain, sleep, fatigue, nausea, and vomiting; Timing of Outcome Measurement (T) was the duration of the intervention; setting of the study (S) was hospital, hospice center or community; and study design (SD) included randomized controlled trials (RCT) or non-randomized controlled trial (NRCT). The exclusion criteria for the articles were as follows: studies whose subjects were not cancer patients or whose interventional technique was not foot reflexology, studies that did not have a comparative intervention, and studies in which the mean and standard deviation of the measured results could not be calculated, as well as case studies, descriptive studies, observational studies, meta-analyses, and review articles rather than experimental studies.

\section{Literature Search}

The data were from articles published until April 30, 2020 from the year when individual electronic databases provided data. The electronic databases that were used for literature search included Pubmed, Cumulative Index to Nursing and Allied Health Literature (CINAHL), Cochrane Central Register of Controlled Trials (CENTRAL), Excerpta Medica Database (EM-BASE), Koreanstudies Information Service System (KISS), KoreaMed, and Research Information Sharing Service (RISS). For the search formula, MeSH terms and text words of the title and abstract were properly applied. The keywords that were used for the search were massage, reflexology, reflexotherapy, relaxation therapy, complementary, alternative, foot, cancer, neoplasm, neoplasia, tutor, malignancy, randomized controlled trial, controlled, trial, groups, placebo, and randomly.

\section{Data Extraction}

In the first stage of the study, data were selected by searching articles in academic journals and dissertations, and in the second stage, the duplicate data were excluded. In the third stage, the relevant data were selected by applying the inclusion and exclusion criteria through the title and abstract of the article. Then in the fourth stage, the final data were selected by reviewing the full text of the article. The selected articles were recorded using a coding table, which consisted of the author, year of publication, country of publication, number of subjects, age of subjects, eligibility criteria, mode of intervention, frequency of intervention, time of intervention, outcome variable, outcome measurement tool, and mean and standard deviation.

\section{Data Analysis}

The effect size and homogeneity of foot reflexology intervention were analyzed using the RevMan 5.2 program of Cochrane Library. When homogeneity was confirmed, the effect size was calculated with a fixedeffects model, and when heterogeneity was confirmed, it was calculated with a random-effects model. The statistical significance of the effect size was judged through the overall effect test and using a 95\% confidence interval (CI), based on the 5\% significance level. The homogeneity of the study was tested using Higgins I2, and the publication bias was tested using the Funnel plot.

\section{Methodological Quality}

Based on Cochrane Library's systematic review guidelines [16] and previous studies [17], the quality of the articles was assessed for five areas: randomization, blinding of outcome assessment, comparison possibility of participants, incomplete outcome data, and selective reporting. For 
each item, the risk of bias was designated as low, unclear or high.

\section{RESULTS}

\section{Literature Search}

In the first stage, 1,050 articles were retrieved through the databases. In the second stage, 342 articles were selected, excluding the duplicate ones from among the retrieved data. In the third stage, 53 articles were selected based on the inclusion and exclusion criteria, focusing on the title and abstract of the article. In the fourth stage, 18 articles were finally selected by reviewing the full text of the article (Fig. 1).

\section{Study Characteristics}

A look at the distribution of the 18 articles included in the systematic review by year will reveal that 15 articles (83.3\%) were published until 2009, and 3 (16.7\%) were published after 2010. By country, 15 articles (83.3\%) were published in South Korea, 2 (11.1\%) were published in the U.S., and 1 (5.6\%) was published in Taiwan. In terms of study design, only 4 articles (22.2\%) were subjected to RCT. The total number of subjects was 931, 468 in the experimental group and 463 in the control group. As for the frequency of interventions, 10 articles (55.6\%) had one to three sessions, and as for the time of intervention, 7 articles (38.9\%) had a 20-minute intervention. In terms of the intervention provider, the intervention was provided by a nurse trained by an expert in 16 articles, by a foot reflexology expert in 1 article, and by the spouse or family member in 1 article (Table 1).

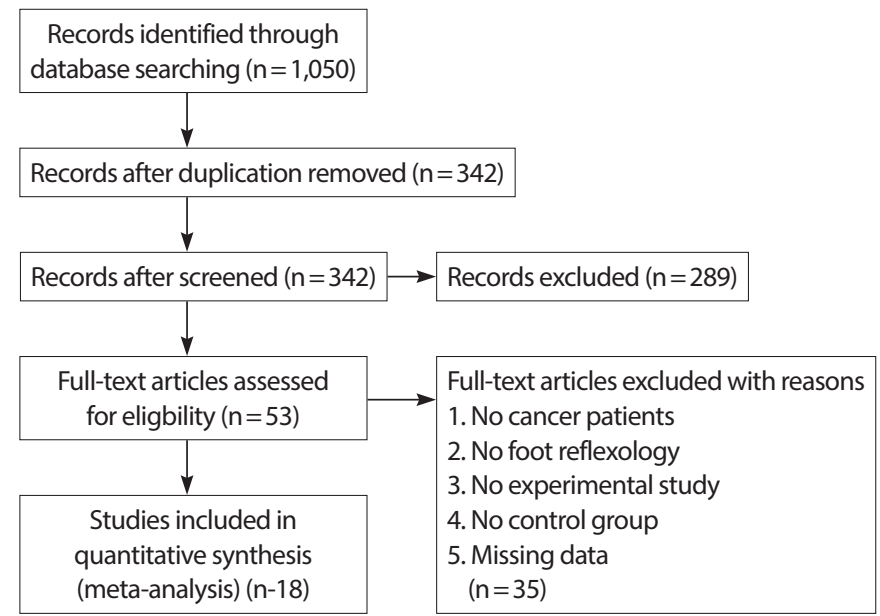

Fig. 1. Flow diagram of the study selection process.

\section{Foot Reflexology Effects}

In eleven articles, foot reflexology was conducted for pain relief. The effect size was -1.05 (95\% CI: -1.52, -0.59), indicating a large effect size, and the heterogeneity was high (I2 =84\%). Seven articles examined the effect on sleep, and the effect size was 1.15 (95\% CI: 0.45, 1.85), showing a large effect size and high heterogeneity $(\mathrm{I} 2=85 \%)$. Six articles examined fatigue, and the effect size was -1.46 (95\% CI: -1.79, -1.14), showing a large effect size and moderate heterogeneity (I2=49\%). Four articles examined nausea and vomiting, and the effect size was -0.53 (95\% CI: $-1.29,0.22$ ), which was not statistically significant (Fig. 2). For the results of the publication bias testing, the risk of publication bias was low in sleep, fatigue and nausea and vomiting, but there was a risk of publication bias in pain.

\section{Methodological Quality}

All the articles had low bias in the comparison possibility of participant and selective reporting. Randomization was conducted by using the random number table or computer program in 3 articles (16.7\%), and the reason for dropout was explained in six articles (33.3\%). Investigator blinding was conducted in 2 articles (11.1\%), showing high bias in this area (Fig. 3).

\section{DISCUSSION}

It was well known that foot reflexology might be a useful non-pharmacological interventions against diverse chemotherapy whereas the scientific lack of evidences. However, there are some references that it can be utilized as a physical and nursing intervention tool for breast cancer patients $[2,5,18]$. Foot reflexology was impacts on fatigue, nausea, and vomiting especially in diverse cancer patients [19]. It was also reported as an effective tools to reduce depression, anxiety, pain and enhance sleep quality and quality of life.

This study investigated the effects of foot reflexology on the physical symptoms of cancer patients by systematically and objectively synthesizing the studies on foot reflexology interventions applied to cancer patients through meta-analysis. For the results of the study, only 3 articles (16.7\%) were published after 2010 among the 18 articles included in the systematic review, and only 3 articles (16.7\%) were published outside of South Korea. Considering that research on other CAMs, such as acupuncture interventions, to alleviate the symptoms of cancer patients has been actively performed of late [20], and that massage therapy is com- 


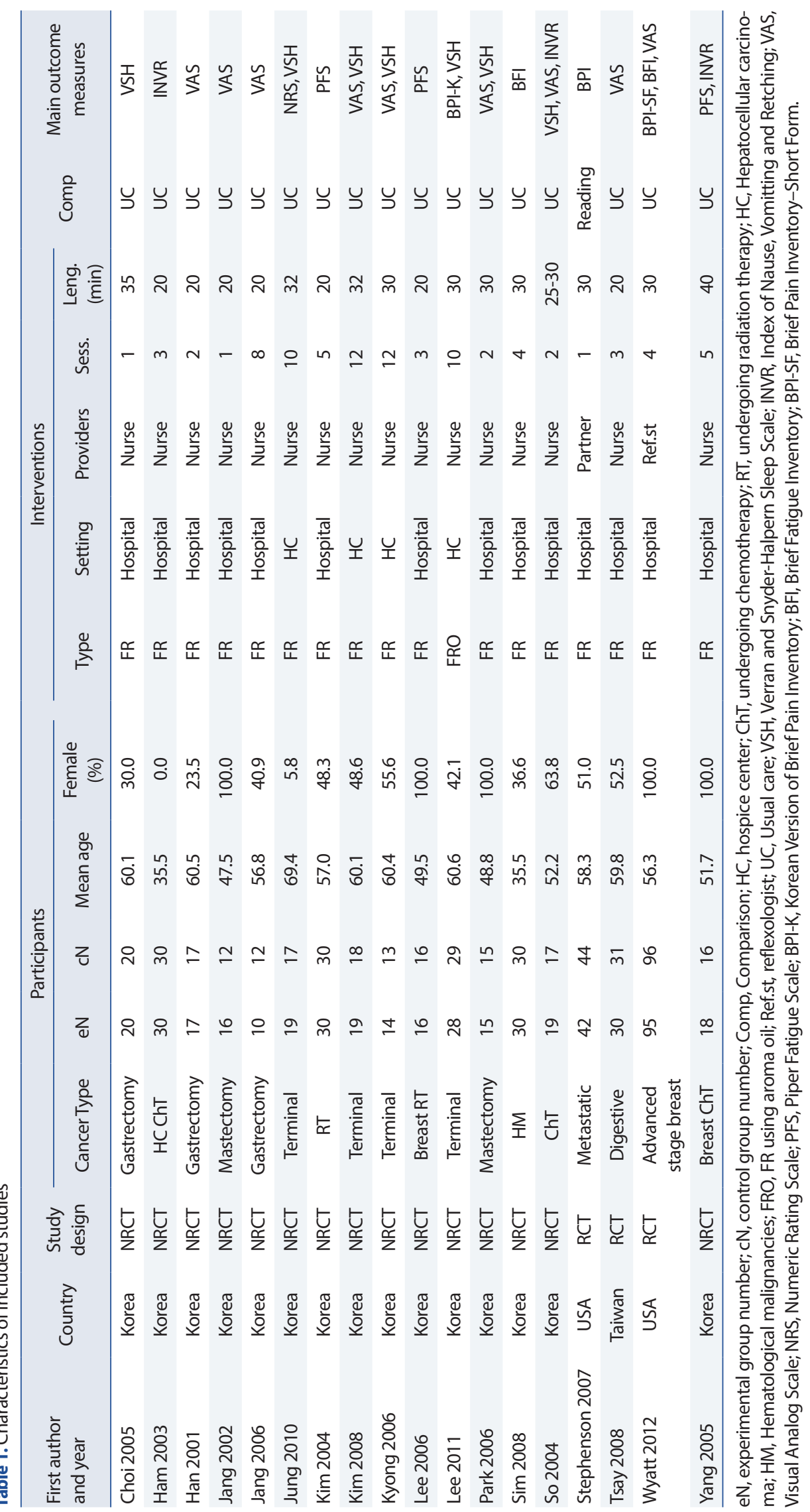




\subsection{Pain}

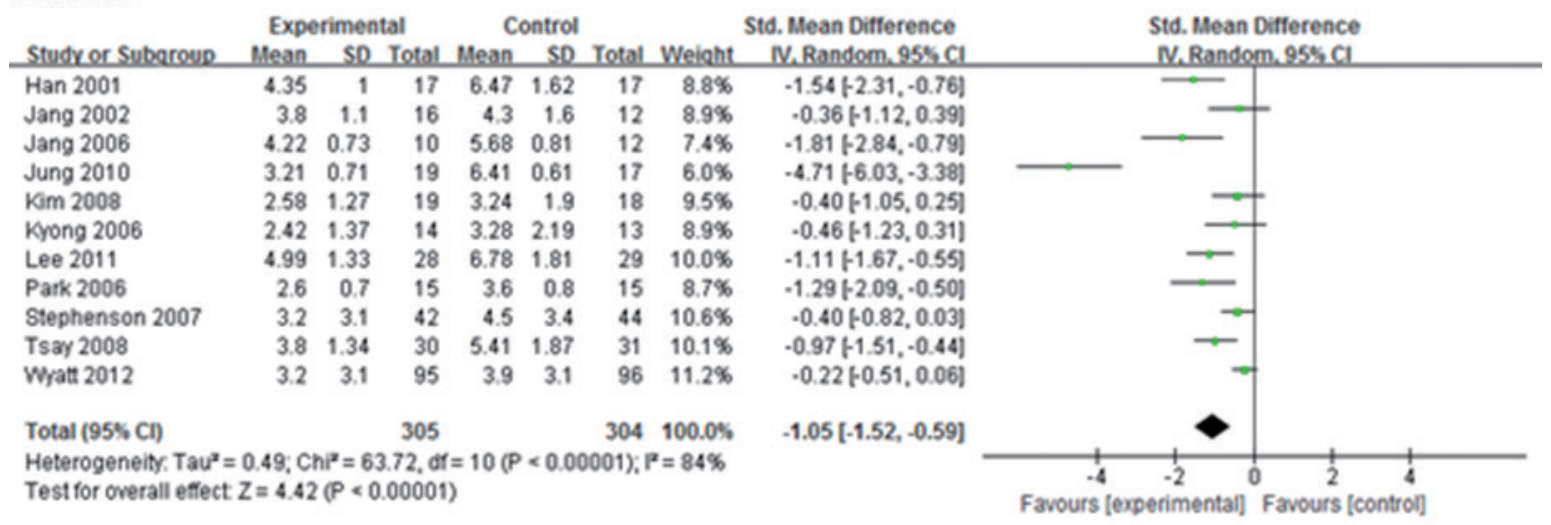

1.2. Sleep

\begin{tabular}{|c|c|c|c|c|c|c|c|}
\hline \multirow[b]{2}{*}{ Studyer Subaroup } & \multicolumn{3}{|c|}{ Experimental } & \multicolumn{2}{|c|}{ Control } & \multirow{2}{*}{\multicolumn{2}{|c|}{ Total Weight }} \\
\hline & Mean & SD & Total & Mean & SD & & \\
\hline Choi 2005 & 35.5 & 5.15 & 20 & 28.1 & 6.22 & 20 & $14.8 \%$ \\
\hline Jung 2010 & 48.37 & 6.45 & 19 & 26.71 & 5.04 & 17 & $12.0 \%$ \\
\hline Kim 2008 & 48.94 & 5.04 & 19 & 45.94 & 5.46 & 18 & $14.9 \%$ \\
\hline Kyong 2006 & 48.86 & 5.05 & 14 & 46 & 6.08 & 13 & $14.2 \%$ \\
\hline Lee 2011 & 39 & 6.45 & 28 & 35.85 & 7.05 & 29 & $15.7 \%$ \\
\hline Park 2006 & 50.6 & 8 & 15 & 37.8 & 3.9 & 15 & $13.4 \%$ \\
\hline 802004 & 44.72 & 15.71 & 19 & 41.33 & 17 & 17 & $14.9 \%$ \\
\hline Total $(95 \% \mathrm{Cl})$ & & & 134 & & & 129 & $100.0 \%$ \\
\hline
\end{tabular}

Std. Mean Difference Std. Mean Difference

IV. Random, $95 \mathrm{~F} \mathrm{Cl}$ IV. Randem, $95 \mathrm{~K} \mathrm{Cl}$

$1.27[0.58,1.96]$

$3.63[2.53,4.74]$

$0.56[-0.10,1.22]$

$0.50+0.27,1.27$

$0.46[-0.07,0.99]$

$1.98[1.08,2.87]$

$0.20[-0.45,0.86]$

$1.15[0.45,1.85]$

Test for overall effect $Z=3.22(P=0.001)$

\subsection{Fatigue}

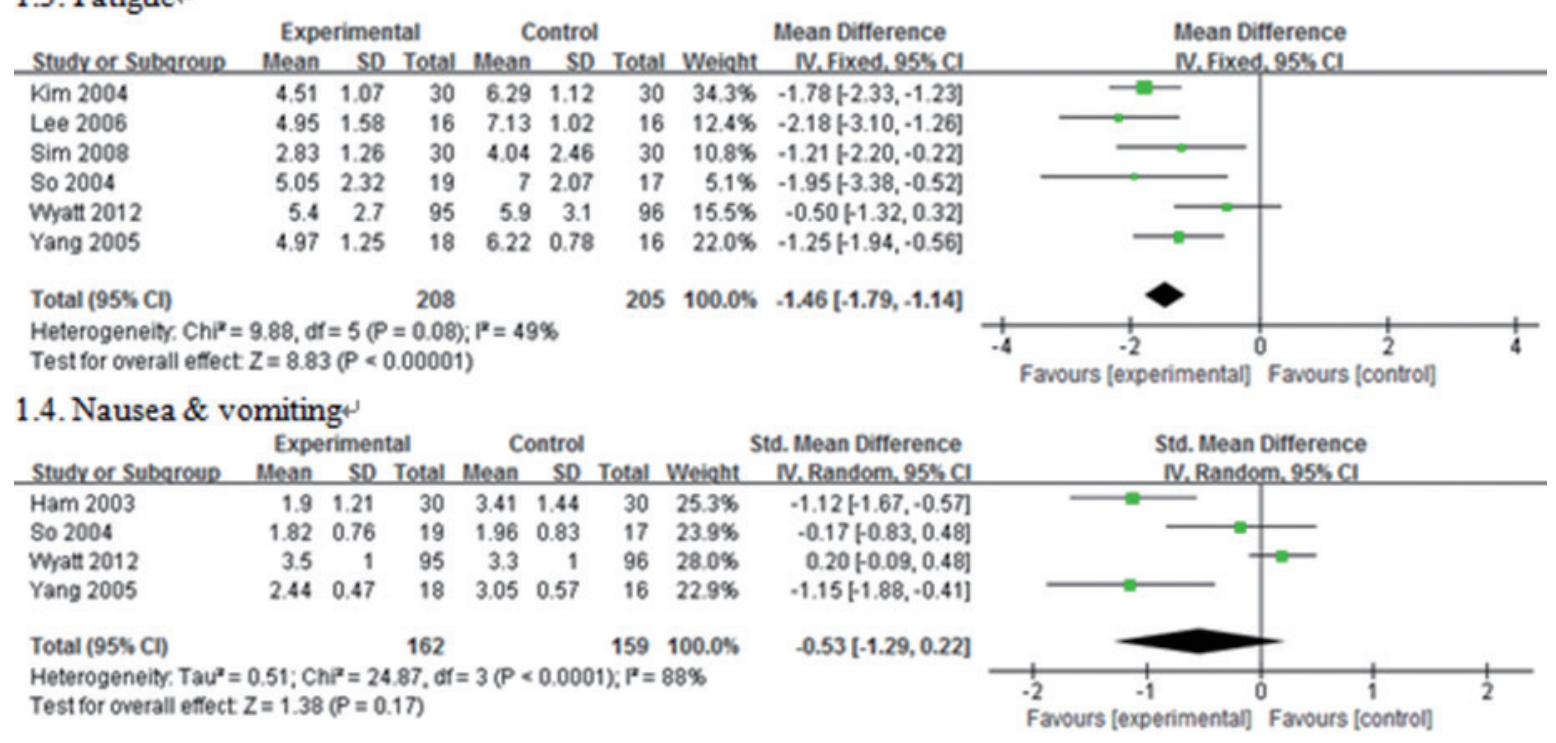

Fig. 2. Forest plot of effect size by foot reflexology on physical symptoms in cancer patients.

monly used at present as a self-care strategy for cancer patients [21], more research on foot reflexology needs to be conducted internationally.

The average intervention time was 27.1 minutes, and the intervention was conducted for one to three sessions per week in 10 articles. This result is similar to the result of the study of Lee et al. [13] that foot reflexology has a shorter intervention time but a moderate effect than other non-pharmacological interventions $[13,14]$. The foot is the most sensitive area for reflex stimulation among the body parts as capillaries, sweat glands, and nerves are concentrated therein. Stimulation of the foot, which has corresponding lymphatic system or body organ reflex points, in spite of only a short intervention, promotes blood circulation in the body through the activation of the parasympathetic nerves, increases the oxygen saturation of the tissues, and promotes detoxification, thereby having a positive effect on physical symptoms of cancer patients [22,23]. 


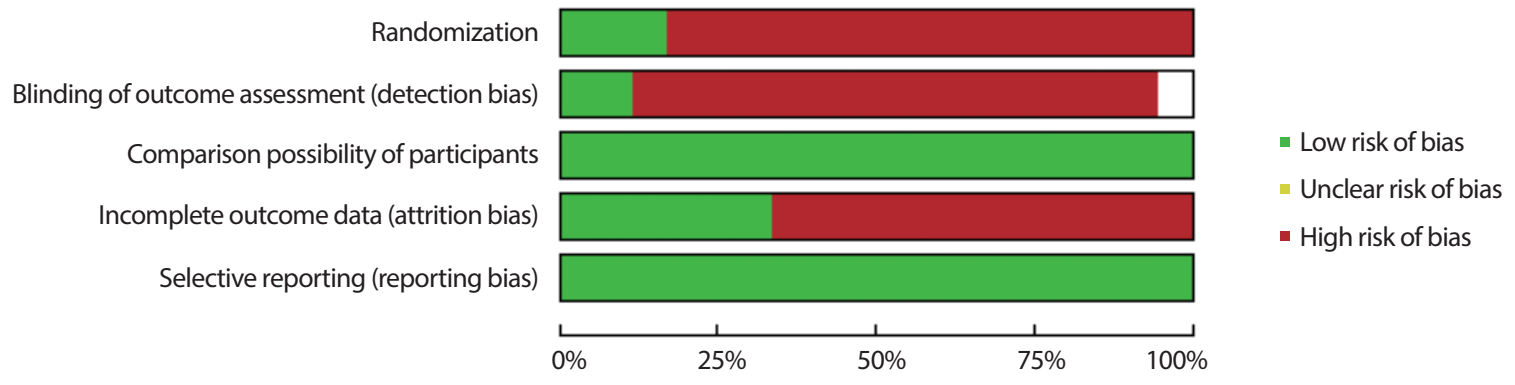

Fig. 3. Assessment of methodological quality.

A look at the items with a high risk of bias in the methodological quality assessment of the articles included in the systematic review will reveal that randomization was not carried out in about $77.8 \%$ of the articles, and that investigator or subject blinding was not conducted in $88.9 \%$ of the articles. For the identification of the net effect of a foot reflexology intervention program, a description of the protocol, which is an important component of intervention fidelity, or use of double blinding, is necessary [24]. Therefore, in future studies, high-quality research should be attempted by describing the details of randomization and explaining the subject and investigator management from the time of the study design.

The meta-analysis revealed that foot reflexology had the greatest effect on fatigue $(\mathrm{d}=-1.46)$. In a study by Kim and Oh [8], a meta-analysis of five fatigues studies measured by Visual Analogue Scale (VAS) or Revised Piper Fatigue Scale (RPFS), foot massage intervention reported a large effect size $(\mathrm{d}=-1.43)$ on fatigue reduction, supporting the result of the present study. Cancer-related fatigue is the most common symptom experienced by up to $80 \%$ of cancer patients [25]. According to the $\mathrm{Na}$ tional Comprehensive Cancer Network ( $\mathrm{NCCN})$ guidelines, the fatigue experienced by cancer patients is independent of physical activity and is characterized by persistent fatigue that is not resolved by rest or sleep [26]. The results of the present study suggest that foot reflexology can be used as a complementary intervention for cancer patients' fatigue management considering that it showed a large fatigue reduction effect in cancer patients.

Foot reflexology also had a large effect on sleep following fatigue $(\mathrm{d}=1.15)$. A meta-analysis study by Lee et al. [13] showed a 1.31 effect size when it was applied to general patients, and a 1.09 effect size in elderly people, showing a similar result as the present study. Sleep disorder was measured by VAS and Korean Sleep Scale and foot massage was done by reflection and relaxation points with pre stage and main stages during 30 minutes [13].
The prevalence of sleep disorder was shown to be twofold higher in cancer patients than in the general population [27], and was shown to be associated with pain and depression, poor quality of life, immune dysfunction, decreased cognitive function, and increased mortality $[28,29,30]$. As the results of the present study confirmed that foot reflexology has a large effect on sleep, it is necessary to apply foot reflexology that is non-invasive and that has a systemic relaxation effect to enhance sleep in the future clinical practice.

To date, the most effective method of controlling pain in cancer patients is known to be the administration of narcotic analgesics. Narcotic analgesics, however, have side effects (e.g., mental confusion, constipation, respiratory depression, nausea and vomiting, and drug addiction) requiring other alternative interventions [31]. The results of this study showed that foot reflexology has a large effect on pain reduction $(\mathrm{d}=$ 1.05). The subjects of the articles in which the pain variable was examined were cancer patients with severe pain (36.4\%) or terminal cancer patients (54.5\%). According to the gate control theory of pain, contact behaviors like massage have been reported to block the transmission of pain to the brain by activating the pain suppression system in the spinal cord.

It was also reported that foot reflexology enhanced the quality of life, and decreased anxiety and pain intensity as well as side effects reduction in stage of metastatic cancer patients $[18,32,33]$. It can deduced a conclusion from the enhanced psychological wellbeing and reduced muscle tension, caused by blood redistribution to the target organ [30,31,33].

This study is significant in that it is the first study to have analyzed the effect size of foot reflexology on the physical symptoms of cancer patients, which it did by systematically reviewing and analyzing the relevant studies conducted at home and abroad. Moreover, it provided a basis for the application of foot reflexology as a complementary and alternative medicine modality for cancer patients. On the other hand, this study also has limitations. First, the information on cultural areas using 
different languages may have been excluded because only the articles published in English and Korean were reflected in the systematic review process. Second, as the meta-analysis was performed using the statistics obtained immediately after the intervention to evaluate the effect of foot reflexology, the long-term effects of foot reflexology could not be examined. Third, there were not many articles providing the statistics necessary for analyzing the effect size, and the heterogeneity of the effect size was high. Thus, the effect size may have been overestimated or underestimated, and care should be taken in interpreting it.

\section{CONCLUSION}

This study systematically reviewed domestic and international studies on foot reflexology, and analyzed the effect of foot reflexology on the physical symptoms of cancer patients. As a result, foot reflexology was found to have a positive effect on pain, sleep, and fatigue in cancer patients. Considering that the number of cancer patients is steadily increasing, it is suggested that foot reflexology be actively used as a complementary and alternative medicine (CAM) modality that can be easily accessed by the general public, rather than limiting the management of the physical symptoms of cancer patients to hospital treatment or drug therapy. To enhance the clinical significance of foot reflexology, well-tailored various meta-analyses should be performed based on the evidence accumulated through subsequent repeated intervention studies.

\section{CONFLICT OF INTEREST}

The authors declare no conflict of interest.

\section{AUTHOR CONTRIBUTIONS}

Conceptualization, YR Yeun, HY Kim, and YS Kwak; Methodology, YR Yeun, and YS Kwak; Formal analysis, YR Yeun, HY Kim, and YS Kwak; Writing-original draft preparation, YR Yeun; Writing-review and editing, HY Kim, and YS Kwak All authors have read and agreed to the published version of the manuscript.

\section{ORCID}

$\begin{array}{ll}\text { Young-Ran Yeun } & \text { https://orcid.org/0000-0002-5393-4312 } \\ \text { Yi-Sub Kwak } & \text { https://orcid.org/0000-0003-4545-7250 }\end{array}$

Hye-Young Kim

https://orcid.org/0000-0001-8511-0601

\section{REFERENCES}

1. Cancer prevalence. National Cancer Information Center: https://www. cancer.go.kr/lay1/S1T639C640/contents.do (accessed 10 August 10 2020).

2. Šeparović R, Silovski T, Tecić Vuger A, Bajić Ž, Silovski H, et al. Association of breast cancer symptoms with patients' quality of life and depression: a croatian cross-sectional study. Psychiatr Danub. 2019;31:928 .

3. Gartland N, Long H, Skevington SM. Undiagnosed cancer symptoms in the community: does poor quality of life influence the decision to seek help? Qual Life Res. 2019;28:1327-35

4. Kim, M.Y. Transition of symptoms and quality of life in cancer patients on chemotherapy. JKAN. 2009;39:433-45.

5. Castanhel FD, Liberali R. Mindfulness-based stress reduction on breast cancer symptoms: systematic review and meta-analysis. Einstein (Sao Paulo). 2018;16:eRW4383.

6. Pedersen AF, Forbes L, Brain K, Hvidberg L, Wulff CN et al. Negative cancer beliefs, recognition of cancer symptoms and anticipated time to help-seeking: an international cancer benchmarking partnership (ICBP) study. BMC Cancer. 2018;18:363.

7. Complementary and alternative medicine. National cancer institute: https://www.cancer.gov/about-cancer/treatment/cam (accessed August $10,2020)$.

8. Kim MY, Oh PJ. Meta-analysis of the effectiveness on foot-reflexomassage for cancer patients. AON. 2011;11:127-35.

9. Wilkinson S, Lockhart K, Gambles M, Storey L. Reflexology for symptom relief in patients with cancer. Cancer Nursing. 2008;31:354-60.

10. Uysal N, Kutluturkan S, Ugur I. Effects of foot massage applied in two different methods on symptom control in colorectal cancer patients: randomized control trial. Int J Nurs. Pract 2017;23.

11. Bahrami T, Rejeh N, Heravi-Karimooi M, Tadrisi SD, Vaismoradi M. The effect of foot reflexology on hospital anxiety and depression in female older adults: a randomized controlled trial. Int J Ther Massage Bodywork. 2019;12:16-21.

12. Zorba P, Ozdemir L. The preliminary effects of massage and inhalation aromatherapy on chemotherapy-induced acute nausea and vomiting: a quasi-randomized controlled pilot trial. Cancer Nurs. 2018;41: $359-66$. 
13. Lee JS, Han MS, Chung YH, Kim J S, Choi JS. Effects of foot reflexology on fatigue, sleep and pain. JKAN. 2011;41:821-33.

14. Lee SH, Kim JY, Yeo S, Kim SH, Lim S. Meta-analysis of massage therapy on cancer pain. Integr Cancer Ther. 2015;14:297-304.

15. Min YC, Oh BJ. A meta-analysis of intervention studies on cancer pain. AON. 2011;11:83-92.

16. Cochrane Methodology Reviews. Cochrane: https://methodology.cochrane.org/welcome (accessed March 1, 2020).

17. Ezzo J, Vickers A, Richardson MA, Allen C, Dibble SL et al. Acupuncture-point stimulation for chemotherapy-induced nausea or vomiting. J Clin Oncol. 2005;23:7188-98.

18. Murat-Ringot A, Jean Souquet P, Chauvenet M, Rentler C, Subtil F et al. The effects of foot reflexology on chemotherapy-induced nausea and vomiting in patients with digestive system or lung cancer: protocol for a randomized controlled trial. JMIR Res Protoc. 2020;14(9):e17232.

19. Yang JH. The effects of foot reflexology on nausea, vomiting and fatigue of breast cancer patients undergoing chemotherapy. Taehan Kanho Kakhoe Chi. 2005;35(1):177.

20. Oh BJ, Kim YL. Effectiveness of non-pharmacologic interventions in chemotherapy induced peripheral neuropathy-a systematic review and meta-analysis. JKAN. 2018;48:123-42.

21. Derksen TM, Bours MJ, Mols F, Weijenberg MP. Lifestyle-related factors in the self-management of chemotherapy-induced peripheral neuropathy in colorectal cancer: a systematic review. Evid Based Complement Alternat Med. 2017;7916031

22. Wilkinson S, Lockhart K, Gambles M, Storey L. Reflexology for symptom relief in patients with cancer. Cancer Nursing. 2008;31:354-60.

23. Armijo-Olivo S, Stiles CR, Hagen NA, Biondo PD, Cummings GG. Assessment of study quality for systematic reviews: a comparison of the cochrane collaboration risk of bias tool and the effective public health practice project quality assessment tool: methodological research. J Eval Clin Pract. 2012;18:12-8

24. Berger AM, Mooney K, Alvarez-Perez A, Breitbart WS, Carpenter KM et al. Cancer-related fatigue. J Natl Compr Canc Netw. 2015;13:101239.

25. Feng L, Chen MK, Lukkahatai N, Hsiao C, Kaushal A et al. Clinical predictors of fatigue in men with non-metastatic prostate cancer receiving external beam radiation therapy. Clin J Oncol Nurs. 2015;19: 744-50.

26. Sleep disorders (PDQ). National Cancer Institute: https://www.cancer gov/about-cancer/treatment/side-effects/sleep-disorders-pdq (accessed August 15, 2020).

27. Garland SN, Johnson JA, Savard J, Gehrman P, Perlis M et al. Sleeping well with cancer: a systematic review of cognitive behavioral therapy for insomnia in cancer patients. Neuropsychiatric Dis Treat. 2014 10:1113-24.

28. Rothrock NE, Cook KF, O'Connor M, Cella D, Smith AW et al. Establishing clinically-relevant terms and severity thresholds for patient-reported outcomes measurement Information system ${ }^{\circledR}\left(\right.$ PROMIS $\left.^{\circledR}\right)$ measures of physical function, cognitive function, and sleep disturbance in people with cancer using standard setting. Qual Life Res. 2019.

29. Steindorf K, Clauss D, Tjaden C, Hackert T, Herbolsheimer F et al. Quality of life, fatigue, and sleep problems in pancreatic cancer patients-A randomized trial on the effects of exercise. Dtsch Arztebl Int. 2019;116:471-8.

30. Asthana R, Goodall S, Lau J, Zimmermann C, Diaz PL et al. Framing of the opioid problem in cancer pain management in Canada. Curr Oncol. 2019; 26:e410-3.

31. McRee LD, Noble S, Pasvogel A. Using massage and music therapy to improve postoperative outcomes. AORN J. 2003;78:433-42.

32. Mendell LM. Constructing and deconstructing the gate theory of pain. Pain. 2014;155:210-6.

33. Braz J, Solorzano C, Wang X, Basbaum AI. Transmitting pain and itch messages: a contemporary view of the spinal cord circuits that generate gate control. Neuron. 2014;82:522-36. 